\title{
DIOPHANTINE APPROXIMATION BY CONTINUED FRACTIONS
}

\author{
JINGCHENG TONG
}

(Received 29 November 1989; revised 3 May 1990)

Communicated by J. H. Loxton

\begin{abstract}
Let $\xi$ be an irrational number with simple continued fraction expansion

$$
\xi=\left[a_{0} ; a_{1}, \ldots, a_{i}, \ldots\right],
$$

$p_{i} / q_{i}$ be its $i$ th convergent. Let $M_{i}=\left[a_{i+1} ; a_{i}, \ldots, a_{1}\right]+\left[0 ; a_{i+2}, a_{i+3}, \ldots\right]$. In this paper we prove that $M_{n-1}<r$ and $M_{n}<R$ imply $M_{n+1}>1 /\left(r^{-1}+a_{n+1} \sqrt{1-4 /(r R)}-a_{n+1}^{2} R^{-1}\right)$, which generalizes a previous result of the author.
\end{abstract}

1980 Mathematics subject classification (Amer. Math. Soc.) (1985 Revision): 11 J 04, 11 A 55.

\section{Introduction}

Let $\xi$ be an irrational number with simple continued fraction expansion $\xi=\left[a_{0} ; a_{1}, \ldots, a_{i}, \ldots\right]$, and $p_{i} / q_{i}$ be its $i$ th convergent. Let $M_{i}=$ $\left[a_{i+1} ; a_{i}, \ldots, a_{1}\right]+\left[0 ; a_{i+2}, a_{i+3}, \ldots\right]$. In a recent paper [9], the present author proved the following conjugate property of the triplet $\left(M_{n-1}, M_{n}, M_{n+1}\right)$, which implies the classical results of Borel and Segre [5] on a symmetric and a symmetric Diophantine approximations.

THEOREM 1. Let $r>a_{n+1}$. Then

(i) $M_{n}>r$ implies $\min \left(M_{n-1}, M_{n+1}\right)<4 r /\left(r^{2}-a_{n+1}^{2}\right)$;

(ii) $M_{n}<r$ implies $\max \left(M_{n-1}, M_{n+1}\right)>4 r /\left(r^{2}-a_{n+1}^{2}\right)$;

(C) 1991 Australian Mathematical Society 0263-6115/91\$A2.00+0.00 
(iii) $M_{n}=r$ implies

$$
\min \left(M_{n-1}, M_{n+1}\right)<4 r /\left(r^{2}-a_{n+1}^{2}\right)<\max \left(M_{n-1}, M_{n+1}\right) .
$$

The essence of Theorem 1 is using the magnitude of $M_{n}$ to estimate the magnitudes of $M_{n-1}$ and $M_{n+1}$. It is very natural to pose two related questions: how to use $M_{n-1}, M_{n+1}$ to estimate $M_{n}$ and how to use $M_{n-1}$, $M_{n}$ to estimate $M_{n+1}$ ? Apparently these questions are more complicated because there are two parameters involved.

In this paper we solve these two questions and show that Theorem 1 is a corollary of our results.

\section{Preliminaries}

Since

$$
M_{i}=\left[a_{i+1} ; a_{i}, \ldots, a_{1}\right]+\left[0 ; a_{i+2}, a_{i+3}, \ldots\right],
$$

letting $P=\left[a_{n+2} ; a_{n+3}, \ldots\right]$ and $Q=\left[a_{n} ; a_{n-1}, \ldots, a_{1}\right]$, we have

$$
\begin{aligned}
& M_{n-1}=Q+\frac{1}{a_{n+1}+P^{-1}}, \\
& M_{n}=a_{n+1}+P^{-1}+Q^{-1},
\end{aligned}
$$

and

$$
M_{n+1}=P+\frac{1}{a_{n+1}+Q^{-1}} .
$$

It is well known $[2,3,4]$ that

$$
\xi-\frac{p_{i}}{q_{i}}=\frac{(-1)^{i}}{M_{i} q_{i}^{2}} .
$$

\section{Main results}

THEOREM 2. Let $\xi$ be an irrational number such that

$$
\xi=\left[a_{0} ; a_{1}, \ldots, a_{i}, \ldots\right] \text {. }
$$

If $r, R$ are two real numbers such that $r>1, R>1$ and $r R>4$, then $M_{n-1}<r$ and $M_{n}<R$ imply

$$
M_{n+1}>\frac{1}{\frac{1}{r}+a_{n+1} \sqrt{1-\frac{4}{R r}}-\frac{a_{n+1}^{2}}{R}} .
$$


Proof. Since $M_{n-1}<r$, by (1) we have $Q^{-1}>1 /\left(r-1 /\left(a_{n+1}+P^{-1}\right)\right)$. By (2) we have

$$
\begin{gathered}
R>a_{n+1}+P^{-1}+\frac{1}{r-\frac{1}{a_{n+1}+P^{-1}}}=\frac{r\left(a_{n+1}+P^{-1}\right)^{2}}{r\left(a_{n+1}+P^{-1}\right)-1}, \\
r\left(a_{n+1}+P^{-1}\right)^{2}-\operatorname{Rr}\left(a_{n+1}+P^{-1}\right)+R<0, \\
a_{n+1}+P^{-1}<\frac{1}{2}\left(R+\sqrt{R^{2}-\frac{4 R}{r}}\right),
\end{gathered}
$$

and

$$
P>2 /\left(R+\sqrt{R^{2}-\frac{4 R}{r}}-2 a_{n+1}\right) .
$$

From $M_{n}<R$ and (2) we have

$$
a_{n+1}+Q^{-1}<R-P^{-1} \text {. }
$$

By (3), we then have

$$
M_{n+1}>P+\frac{1}{R-P^{-1}} .
$$

By (7), we have $R P>P\left(a_{n+1}+P^{-1}+Q^{-1}\right)>a_{n+1} P+1>2$.

This implies that the right-hand side of $(8)$ is an increasing function of $P$. By (6) and (8) we then have

$$
M_{n+1}>1 /\left(\frac{1}{r}+a_{n+1} \sqrt{1-\frac{4}{R r}}-\frac{a_{n+1}^{2}}{R}\right) .
$$

REMARK 1. In Theorem 2, if we reverse the directions of the inequality signs in the proof, we have a conjugate theorem.

TheOREM $2^{\prime}$. Let $\xi, r, R$ be given as in Theorem 2. Then $M_{n-1}>r$ and $M_{n}>R$ imply

$$
M_{n+1}<1 /\left(\frac{1}{r}+a_{n+1} \sqrt{1-\frac{4}{R r}}-\frac{a_{n+1}^{2}}{R}\right) .
$$

RemarK 2. The conditions $M_{n-1}<r$ and $M_{n}<R$ in Theorem 2 can be changed to be $M_{n-1} \leq r$ and $M_{n} \leq R$ but $M_{n-1}=r$ and $M_{n}=R$ do not hold simultaneously. A similar result is true for Theorem $2^{\prime}$. 
REMARK 3. If we interchange the roles of $M_{n+1}$ and $M_{n-1}$ in the proof of Theorem 2, and use equation (3) instead of equation (1), the conclusion of the theorem becomes $M_{n}<R$ and $M_{n+1}<r$ implying

$$
M_{n-1}>1 /\left(\frac{1}{r}+a_{n+1} \sqrt{1-\frac{4}{R r}}-\frac{a_{n+1}^{2}}{R}\right) .
$$

As in Remark 2, the inequalities $M_{n}<R$ and $M_{n+1}<r$ may be replaced by $M_{n} \leq R$ and $M_{n+1} \leq r$, provided that $M_{n}=R$ and $M_{n+1}=r$ do not hold simultaneously.

COROLlaRy 1. Let $r, r^{\prime}$ be two real numbers such that $r>1, r^{\prime}>1$. Then $M_{n-1}<r$ and $M_{n+1}<r^{\prime}$ imply

$$
M_{n}>\frac{\frac{1}{r}+\frac{1}{r^{\prime}}+\sqrt{a_{n+1}^{2}+\frac{4}{r r^{\prime}}}}{1-a_{n+1}^{-2}\left(\frac{1}{r}-\frac{1}{r^{\prime}}\right)^{2}}
$$

Proof. Let $H$ be the right-hand side of inequality (9). It is easy to check the following equality.

$$
\left(a_{n+1}^{-2}\left(\frac{1}{r}-\frac{1}{r^{\prime}}\right)^{2}-1\right) H^{2}+2\left(\frac{1}{r}+\frac{1}{r^{\prime}}\right) H+a_{n+1}^{2}=0
$$

We consider two possible cases.

CASE 1. $r \leq r^{\prime}$. Rewrite (10) as follows:

$$
\left(a_{n+1}^{-1}\left(\frac{1}{r}-\frac{1}{r^{\prime}}\right) H+a_{n+1}\right)^{2}=H^{2}-\frac{4 H}{r^{\prime}} .
$$

Since $r>1, r^{\prime}>1$, we have $0<1-a_{n+1}^{-2}\left(1 / r-1 / r^{\prime}\right)^{2} \leq 1$. From $r \leq r^{\prime}$ we have

$$
r^{\prime}\left(\frac{1}{r}+\frac{1}{r^{\prime}}+\sqrt{a_{n+1}^{2}+\frac{4}{r r^{\prime}}}\right)>\frac{r^{\prime}}{r}+1+\sqrt{\frac{4 r^{\prime}}{r}} \geq 4 .
$$

Hence $H r^{\prime}>4$ and (11) becomes

$$
\begin{gathered}
a_{n+1}^{-1}\left(\frac{1}{r}-\frac{1}{r^{\prime}}\right) H+a_{n+1}=H \sqrt{1-\frac{4}{H r^{\prime}}}, \\
r=1 /\left(\frac{1}{r^{\prime}}+a_{n+1} \sqrt{1-\frac{4}{H r^{\prime}}}-\frac{a_{n+1}^{2}}{H}\right) .
\end{gathered}
$$


If $M_{n} \leq H$, by $M_{n+1}<r^{\prime}$ and Remark 3, we have

$$
M_{n-1}>1 /\left(\frac{1}{r^{\prime}}+a_{n+1} \sqrt{1-\frac{4}{H r^{\prime}}}-\frac{a_{n+1}^{2}}{H}\right)=r \text {, }
$$

contradicting the assumption that $M_{n-1}<r$. Hence $M_{n}>H$.

CASE 2. $r>r^{\prime}$. By a similar method we can prove that

$$
r^{\prime}=1 /\left(\frac{1}{r}+a_{n+1} \sqrt{1-\frac{4}{H r}}-\frac{a_{n+1}^{2}}{H}\right) .
$$

By Theorem 2, $M_{n} \leq H$ and $M_{n-1}<r$ imply $M_{n+1}>r^{\prime}$, contradicting the assumption that $M_{n+1}<r^{\prime}$. Hence $M_{n}>H$.

Now we discuss a special case of Corollary 1. By (1) and (3), if $M_{n-1}=$ $M_{n+1}$ we have $(P-Q)\left(a_{n+1} P Q+P+Q\right)=0$. Hence $P=Q$. But $P$ is an infinite continued fraction, and hence irrational, while $Q$ is finite and hence rational so that $P \neq Q$. Therefore $M_{n-1}=r$ and $M_{n+1}=r$ cannot hold simultaneously. Corollary 1 may be varied in the same way as Theorem 1 , as described in Remarks 1,2 and 3. By these variations and setting $r=r^{\prime}$, we obtain the following result.

COROLlaRy 2. Let $r>1$ be a real number. Then

(i) $M_{n-1} \leq r$ and $M_{n+1} \leq r$ imply

$$
M_{n}>\frac{2}{r}+\sqrt{a_{n+1}^{2}+\frac{4}{r^{2}}}
$$

(ii) $M_{n-1} \geq r$ and $M_{n+1} \geq r$ imply

$$
M_{n}<\frac{2}{r}+\sqrt{a_{n+1}^{2}+\frac{4}{r^{2}}} .
$$

REMARK 4. Theorem 1 is a simple corollary to Corollary 2 because $\min \left(M_{n-1}, M_{n+1}\right) \geq 4 r /\left(r^{2}-a_{n+1}^{2}\right)$ implies, by (ii), that $M_{n}<r$ and $\max \left(M_{n-1}, M_{n+1}\right) \leq 4 r /\left(r^{2}-a_{n+1}^{2}\right)$ implies, by (i), that $M_{n}>r$. The contrapositives of these two statements imply the three parts of Theorem 1 .

We give another application of Corollary 2 .

In [7], the present author proved that if $\tau$ is a real number such that $1 \leq \tau<2+\sqrt{5}$, then among any three consecutive convergents $p_{i} / q_{i} \quad(i=$ $n-1, n, n+1)$ of an irrational number $\xi$, at least one satisfies the following inequality:

$$
\frac{-1}{\sqrt{a_{n+1}^{2}+4 \tau} \quad q_{i}^{2}}<\xi-\frac{p_{i}}{q_{i}}<\frac{\tau}{\sqrt{a_{n+1}^{2}+4 \tau} \cdot q_{i}^{2}} .
$$


We show that the restriction $\tau<2+\sqrt{5}$ may be dropped.

TheORem 3. Let $\tau \geq 1$ be a real number. Then amorig in we chisciuive convergents $p_{i} / q_{i}(i=n-1, n, n+1)$ of an irrational number $\xi$, at least one satisfies inequality (12).

Proof. By (4) we need only prove that either there is an odd index $i$ among $n-1, n, n+1$ such that $M_{i}>\sqrt{a_{n+1}^{2}+4 \tau}$, or there is an even index $i$ such that $M_{i}>\sqrt{a_{n+1}^{2}+4 \tau} / \tau$.

We discuss two possible cases.

CASE 1. $n$ is odd. Then $n-1$ and $n+1$ are even. If one of $M_{n-1}, M_{n+1}>$ $\sqrt{a_{n+1}^{2}+4 \tau} / \tau$, then (12) holds by (4). If both $M_{n-1}, M_{n+1} \leq \sqrt{a_{n+1}^{2}+4 \tau} / \tau$, we may assume $\sqrt{a_{n+1}^{2}+4 \tau} / \tau>1$ because $\sqrt{a_{n+1}^{2}+4 \tau} / \tau \leq 1$ implies

$$
M_{n+1}>a_{n+2} \geq 1 \geq \sqrt{a_{n+1}^{2}+4 \tau} / \tau \text {. }
$$

Letting

$$
r=\sqrt{a_{n+1}^{2}+4 \tau} / \tau
$$

in Corollary 2(i), we have $M_{n}>\sqrt{a_{n+1}^{2}+4 \tau}$.

CASE 2. $n$ is even. Then $n-1$ and $n+1$ are odd.

If one of $M_{n-1}, M_{n+1}>\sqrt{a_{n+1}^{2}+4 \tau}$, then (12) holds by (4).

If both $M_{n-1}, M_{n+1} \leq \sqrt{a_{n+1}^{2}+4 \tau}$, then letting $r=\sqrt{a_{n+1}^{2}+4 \tau}>1$ in Corollary 2(i), we have

$$
M_{n}>\frac{2+\sqrt{a_{n+1}^{4}+4 a_{n+1}^{2} \tau+4}}{\sqrt{a_{n+1}^{2}+4 \tau}} .
$$

Since $\tau \geq 1$, the right-hand side of (13) is greater than $\sqrt{a_{n+1}^{2}+4 \tau} / \tau$. The proof is complete.

REMARK 5. An alternative proof of Theorem 3 can be found in [1].

\section{Acknowledgement}

The author thanks the referee sincerely for his valuable suggestions to improve this paper. 


\section{References}

[1] C. Kraaikamp, 'On the approximation by continued fractions', preprint.

[2] W. J. LeVeque, Topics in Number Theory I, II, (Addison-Wesley Publ. Co., 1956).

[3] O. Perron, Die Lehre von den Kettenbruchen I, II, 3rd ed., (Teubner, Leipzig, 1954).

[4] W. M. Schmidt, Diophantine Approximation, (Lecture Notes in Math. 785, SpringerVerlag, 1980).

[5] B. Segre, 'Lattice points in infinite domains and asymmetric Diophantine approximation', Duke J. Math. 12 (1945), 337-365.

[6] J. Tong, 'The conjugate property of the Borel theorem on Diophantine approximation', Math. Z. 184 (1983), 151-153.

[7] _ _ 'A theorem on approximation of irrational numbers by simple continued fractions', Proc. Edinburgh Math. Soc. 31 (1988), 197-204.

[8] _ , 'Segre's theorem on asymmetric Diophantine approximation', J. Number Theory 28 (1988), 116-118.

[9] __ 'The conjugate property for Diophantine approximation of continued fractions', Proc. Amer. Math. Soc. 105 (1989), 535-539.

Department of Mathematics and Statistics

University of North Florida

Jacksonville, Florida 32216

U.S.A. 